\title{
PRIME MAPPINGS
}

\author{
BY \\ L. B. TREYBIG $\left({ }^{1}\right)$
}

1. Introduction. Suppose $A_{n}=\left\{a_{1}, a_{2}, \ldots, a_{2 n}\right\}$ is a set of $2 n$ points lying in the open interval $(0,1)$ such that $a_{i}<a_{i+1}, i=1, \ldots, 2 n-1$ and that $W$ is a decomposition of $A_{n}$ into two element sets. Suppose also that $f$ is a mapping of the half open interval $[0,1)$ into the plane such that (1) $f(t)=f\left(t^{\prime}\right)$ for $t<t^{\prime}$ if and only if $\left\{t, t^{\prime}\right\} \in W$, (2) $\operatorname{Im} f$ can be expressed as the sum of a finite number of straight line intervals such that no point of $f\left(A_{n}\right)$ is an endpoint of one of the intervals and, (3) $f(t) \rightarrow f(0)$ as $t \rightarrow 1$. The decomposition $W$ is said to determine the double point structure of $f$, and $W$ is said to have property $P$ provided it is true that if $U$ and $V$ are subsets of $W$ such that $U=W-V$, then there exist $\left\{u_{1}, u_{2}\right\} \in U$ and $\left\{v_{1}, v_{2}\right\} \in V$ such that $u_{1}<v_{1}<u_{2}<v_{2}$ or $v_{1}<u_{1}<v_{2}<u_{2}$. If $W$ has property $P$ and the double point structure of $f$ is determined by $W$ then $f$ is said to have property $P$ or be prime. It is now possible to state two of the main results.

THeOREM 2 (The INVARIANCE OF Boundary TheOREM). If $A_{n}$ (as above) is a set of $2 n$ points lying in $(0,1), W$ is a decomposition of $A_{n}$ into two element sets and $f$ and $g$ are prime mappings whose double point structure is determined by $W$, then there is a natural one-to-one correspondence between the complementary domains of $\operatorname{Im} f$ and those of $\operatorname{Im} g$ according to the equation $f^{-1}(\mathrm{Bd} U)=g^{-1}(\mathrm{Bd} V)$, where $U$ and $V$ are corresponding complementary domains of $\operatorname{Im} f$ and $\operatorname{Im} g$, respectively.

THEOREM 3. Given $A_{n}, W$, fand $g$ as in Theorem 2 , and assuming that the unbounded complementary domains of $\operatorname{Im} f$ and $\operatorname{Im} \mathrm{g}$ correspond, then there is a homeomorphism $h$ from $E^{2}$ onto $E^{2}$ such that $h f=g$.

The main use of Theorem 3 (to the author), and, certainly the context in which it arose, are now described. Given $f$ as in paragraph one, the set $\operatorname{Im} f$ can be considered [1] as the projection of a polygonal knot in regular position, where the set $f\left(A_{n}\right)$ is the set of double points of the projection. Suppose $g$ is a one-to-one mapping of $[0,1)$ into $E^{3}$ so that (1) $\pi g=f$, where $\pi(x, y, z)=(x, y, 0)$, (2) $\operatorname{Im} g$ is the sum of a finite number of straight line intervals, and (3) $g(t) \rightarrow g(0)$ as $t \rightarrow 1$. D. E. Penney [6] has been studying the idea of associating with $g$ (or $\operatorname{Im} g$ ) a "word" $f\left(a_{1}\right)^{e_{1}} f\left(a_{2}\right)^{e_{2}} \ldots f\left(a_{2 n}\right)^{e_{2 n}}$, where if $f\left(a_{i}\right)=f\left(a_{j}\right)$ and the $z$ coordinate of $g\left(a_{i}\right)$ is larger than the $z$ coordinate of $g\left(a_{j}\right)$, then $e_{i}=1$ (or is suppressed) and $e_{j}=-1$. The technique is illustrated in Figure 1.

Presented to the Society, November 13, 1965; received by the editors April 15, 1966.

(1) This research was partially supported by NSF Grant GP3989. 
In [6] Penney describes a set of "admissible" operations with "words", one example of which is the cancellation of the $a a^{-1}$ in the "word" associated with Figure 1. His Theorem 1 (applied here) says that there is some knot isomorphic to the one of Figure 1 and whose word is $b^{-1} c d^{-1} b e^{-1} d c^{-1} e$. A prime word is one where $\pi g$ is a prime mapping. Penney's Theorem 3 says that if $F$ and $G$ are knots

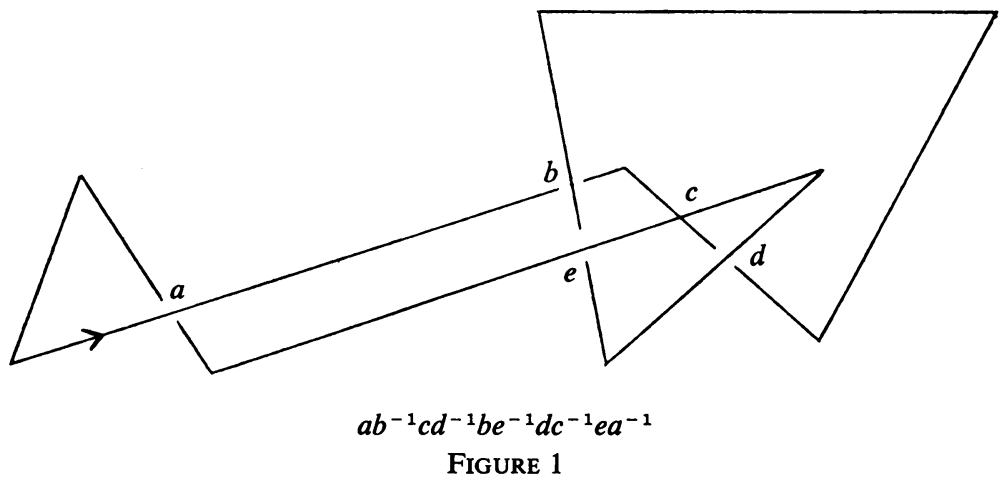

with words $W_{1}$ and $W_{2}$, respectively, $W_{2}$ is prime and can be obtained by a finite number of "admissible" operations on $W_{1}$, then $F$ and $G$ are isomorphic. Theorem 3 of this paper is one of the preliminaries to the Theorem 3 of Penney's paper.

For other references in the field of topology see Gauss [2], Nagy [5] and Treybig [9]. For references in the field of topological analysis see Marx [3] and Titus [7] and [8].

2. Definitions. In addition to the definitions stated in the introduction it is desirable to state a few others. Given $A_{n}$ a subset of $(0,1)$ as above, let $N\left(A_{n}\right)$ denote the set of all mappings $f$ of $[0,1)$ into the plane such that there is a decomposition $W$ of $A_{n}$ into two element sets and such that $f$ and $W$ are related as above. Let $G\left(A_{n}\right)$ denote $\left\{\left[a_{1}, a_{2}\right], \ldots,\left[a_{2 n-1}, a_{2 n}\right],\left[0, a_{1}\right]+\left[a_{2 n}, 1\right)\right\}$. The notation $\left[0, a_{1}\right]+\left[a_{2 n}, 0\right)$ will be shortened to $\left[a_{2 n}, a_{1}\right]$. As in the case of other intervals, the points $a_{2 n}$ and $a_{1}$ will be called the endpoints of this set. Given a collection $H$ of point sets let $H^{*}$ denote the sum (or union) of the sets in $H$. Given a decomposition $W$ of $A_{n}$ as above, then $W$ will be said to have property $Q$ provided it is true that if $\left\{a_{i}, a_{j}\right\} \in W$ then there exists $\left\{a_{r}, a_{s}\right\} \in W$ such that $i<r<j<s$ or $r<i<s<j$. If $W$ determines the double point structure of $f \in N\left(A_{n}\right)$ and $W$ has property $Q$, then $f$ will be said to have property $Q$. It is easy to see that property $P$ implies property $Q$ for $n>1$.

\section{A lemma.}

LEMMA 1. If $f \in N\left(A_{n}\right)$ and each of $A B$ and $C D$ is an arc lying in $\operatorname{Im} f$ such that $\{A, B, C, D\} \subset f\left(A_{n}\right)$, then (1) there exists $H, K$ such that $H \subset G\left(A_{n}\right), K \subset G\left(A_{n}\right)$, $f\left(H^{*}\right)=A B$ and $f\left(K^{*}\right)=C D$, and (2) if $A B$ and $C D$ intersect, then the first (last) point of $C D$ on $A B$ in the order from $A$ to $B$ is in $f\left(A_{n}\right)$. 
Proof. (1) Let $H=\left\{G \in G\left(A_{n}\right): f\left(G-G \cdot A_{n}\right)\right.$ intersects $\left.A B\right\}$. If $f\left(G-G \cdot A_{n}\right)$ intersects $A B$ then it must be a subset of $\operatorname{arc} A B$ or it would contain one of the endpoints $A$ or $B$. Therefore, since $A B$ is closed, $H$ contains a finite number of closed sets and $f\left(H^{*}\right)$ is dense in $A B$, it follows that $f\left(H^{*}\right)=A B$. Define $K$ for $C D$ analogously.

(2) Suppose the first point $X$ of $C D$ on $A B$ in the order from $A$ to $B$ is not in $f\left(A_{n}\right)$. There exists $G \in G\left(A_{n}\right)$ so that $X \in f\left(G-G \cdot A_{n}\right)$ so $G \in H \cdot K$. Thus there is an open interval containing $X$ which is a subset of both $A B$ and $C D$. This yields a contradiction.

\section{The theorems.}

THEOREM 1. If $A_{n}$ (as above) is a set of $2 n$ points lying in $(0,1), W$ is a decomposition of $A_{n}$ into two element sets and f and $g$ are prime mappings whose double point structure is determined by $W$, then for each complementary domain $U$ of $\operatorname{Im} f$ there is a unique complementary domain $V$ of $\operatorname{Im} g$ such that $f^{-1}(\operatorname{Bd} U)=g^{-1}(\operatorname{Bd} V)$.

Proof. By Theorem 7 of [9] the collection $H_{1}$ of all elements $K$ of $G\left(A_{n}\right)$ such that $f\left(K-K \cdot A_{n}\right)$ intersects $\mathrm{Bd} U$ has the property that $f\left(H_{1}^{*}\right)=\mathrm{Bd} U$, and is the only subcollection of $G\left(A_{n}\right)$ with this property. Suppose $W_{1}=\left\{a_{r}, a_{s}\right\} \in W(r<s)$ and that each of $I_{1}$ and $I_{2}$ is an element of $H_{1}$ such that $a_{r}$ is an endpoint of $I_{1}$ and $a_{s}$ is an endpoint of $I_{2}$. (see Theorem 9 of [9]). By Theorem 5 of [9] there is a complementary domain $V$ of $\operatorname{Im} g$ such that $g\left(I_{1}\right)+g\left(I_{2}\right) \subset \mathrm{Bd} V$. Let $H_{2}$ denote the unique subcollection of $G\left(A_{n}\right)$ such that $g\left(H_{2}^{*}\right)=\mathrm{Bd} V$. The idea is to show that $H_{2}=H_{1}$, so suppose that $H_{1} \neq H_{2}$.

If $n=1$ then it follows that $H_{1}=H_{2}$, so the previous assumption means that $n>1$, and that $W$ has property $Q$. By Theorem 9 of [9], (1) $\mathrm{Bd} U(\mathrm{Bd} V)$ is a simple closed curve, and (2) if $a_{i} \in A_{n} \cdot K \subset K \in H_{1}\left(H_{2}\right)$ there is exactly one other element $L$ of $H_{1}\left(H_{2}\right)$ containing an element $a_{j}$ of $A_{n}$ such that $f\left(a_{i}\right)=f\left(a_{j}\right)$, and furthermore $K$ and $L$ do not intersect. With the aid of Lemma $1 H_{1}\left(H_{2}\right)$ can be expressed as the sum of two subcollections $\left\{I_{2}\right\}$ and $\left\{J_{1}, J_{2}, \ldots, J_{m_{1}}\right\}\left(\left\{I_{2}\right\}\right.$ and $\left.\left\{K_{1}, \ldots, K_{m_{2}}\right\}\right)$ such that (1) $f\left(I_{2}\right)$ and $f\left(\sum J_{p}\right)\left(g\left(I_{2}\right)\right.$ and $\left.g\left(\sum K_{p}\right)\right)$ are two arcs which meet only in their endpoints, and (2) $f\left(J_{p}\right)$ intersects $f\left(J_{q}\right)\left(g\left(K_{p}\right)\right.$ intersects $\left.g\left(K_{q}\right)\right)$ if and only if $|p-q| \leqq 1,1 \leqq p, q \leqq m_{1}\left(m_{2}\right),(3) K_{1}=J_{1}=I_{1}$. There is an integer $n_{1}$ such that $J_{p}=K_{p}$ for $1 \leqq p \leqq n_{1}$, but $J_{n_{1}+1} \neq K_{n_{1}+1}$. Furthermore, there is an integer $n_{2}>n_{1}$ such that $f\left(J_{n_{2}}\right)$ intersects $f\left(\sum K_{p}\right)$, but if $n_{1}<q<n_{2}$ then $f\left(J_{q}\right)$ does not intersect $f\left(\sum K_{p}\right)$.

As above $H_{1}\left(H_{2}\right)$ is the sum of two subcollections $A_{1}$ and $A_{2}\left(B_{1}\right.$ and $\left.B_{2}\right)$ such that (1) $\mathrm{Bd} U(\mathrm{Bd} V)$ is the sum of two arcs $f\left(A_{1}^{*}\right)$ and $f\left(A_{2}^{*}\right)\left(g\left(B_{1}^{*}\right)\right.$ and $\left.g\left(B_{2}^{*}\right)\right)$ having only their endpoints in common, (2) $A_{1}=\left\{J_{n_{1}+1}, \ldots, J_{n_{2}}\right\}$, and (3) $f\left(B_{1}^{*}\right)$ has the same end points as $f\left(A_{1}^{*}\right)$ but they have no other point in common.

If $f\left(B_{2}^{*}\right)$ intersects $f\left(A_{1}^{*}\right)$ in a point distinct from one of its endpoints, then $f\left(B_{2}^{*}\right)$ contains an arc $D_{2}$ and $f\left(B_{1}^{*}\right)$ contains an arc $D_{1}$ such that (1) $D_{1}$ and $D_{2}$ do not intersect, (2) $D_{1}$ and $D_{2}$ lie except for their endpoints in the complement 
of $\bar{U}$ and the endpoints of $D_{1}$ separate those of $D_{2}$ on Bd $U$. By Theorem 11, p. 147 of [4], $D_{1}$ and $D_{2}$ must intersect, which is a contradiction. Therefore, the situation is that (1) $f\left(A_{1}^{*}\right) \cdot f\left(A_{2}^{*}\right)=P+Q=f\left(B_{1}^{*}\right) \cdot f\left(B_{2}^{*}\right),(2) f\left(H_{2}^{*}\right) \cdot f\left(A_{2}^{*}\right)=P+Q$ and (3) $f\left(H_{1}^{*}\right) \cdot f\left(B_{2}^{*}\right)=P+Q$, where $P=f\left(a_{i}\right), Q=f\left(a_{p}\right),\left\{a_{i}, a_{j}\right\} \in W,\left\{a_{p}, a_{q}\right\} \in W$, and $a_{i}$ is an endpoint of $J_{n_{1}}=K_{n_{1}}$. It may be supposed without loss of generality that $i<j, p, q$ and that $a_{p}$ is an endpoint of $J_{n_{2}}$. Let $K$ denote the collection of all elements of $G\left(A_{n}\right)$ which have none of $a_{i}, a_{j}, a_{p}$ and $a_{q}$ for an endpoint. The idea now is to try to obtain a subset $L$ of $K$ such that $f\left(L^{*}\right)$ is connected and intersects both of $f\left(A_{1}^{*}\right)$ and $f\left(A_{2}^{*}\right), f\left(A_{1}^{*}\right)$ and $f\left(B_{2}^{*}\right), f\left(A_{2}^{*}\right)$ and $f\left(B_{1}^{*}\right)$, or $f\left(B_{1}^{*}\right)$ and $f\left(B_{2}^{*}\right)$.

Suppose that $J_{n_{1}}=K_{n_{1}}=\left[a_{i-1}, a_{i}\right]$, and note that one of $\left[a_{j-1}, a_{j}\right]$ and $\left[a_{j}, a_{j+1}\right]$ is an element of $A_{2}$ and the other is an element of $B_{2}$.

Case 1. $i<p, q<j$. Let $L=\left\{\left[a_{1}, a_{2}\right], \ldots,\left[a_{i-2}, a_{i-1}\right],\left[a_{j+1}, a_{j+2}\right], \ldots,\left[a_{2 n}, a_{1}\right]\right\}$. ( $i<p, q<j$ here means $i<p<j$ and $i<q<j$.)

Case 2. $i<q, j<p$ and $\left[a_{p}, a_{p+1}\right]$ is an element of $A_{2}$ or $B_{2}$. Let

$$
L=\left[a_{1}, a_{2}\right], \ldots,\left[a_{i-2}, a_{i-1}\right],\left[a_{p+1}, a_{p+2}\right], \ldots,\left[a_{2 n}, a_{1}\right] .
$$

Case 3. $i<p, j<q$ and $\left[a_{q}, a_{q+1}\right]$ is an element of $A_{2}$ or of $B_{2}$ (analogous to 2). Case 4. $i<j, p<q$ and $\left[a_{q}, a_{q+1}\right]$ is not an element of $A_{2}+B_{2}$.

(a) $j<p$. In this case each of the sets

$$
\begin{aligned}
& L_{1}=\left\{\left[a_{i+1}, a_{i+2}\right], \ldots,\left[a_{j-2}, a_{j-1}\right]\right\}, \\
& L_{2}=\left\{\left[a_{j+1}, a_{j+2}\right], \ldots,\left[a_{p-2}, a_{p-1}\right]\right\},
\end{aligned}
$$

and

$$
L_{3}=\left\{\left[a_{p+1}, a_{p+2}\right], \ldots,\left[a_{q-2}, a_{q-1}\right]\right\}
$$

has the property that $f\left(L_{i}^{*}\right)$ is connected $(i=1,2,3)$ and intersects $f\left(A_{2}^{*}\right)$ or $f\left(B_{2}^{*}\right)$. Since $W$ has property $Q$ there is an element $\left\{a_{v}, a_{w}\right\}$ of $W$ such that $i<v<q$ and $w<i$ or $w>q$. Let $L=L_{t}+\left\{\left[a_{q+1}, a_{q+2}\right], \ldots,\left[a_{2 n}, a_{1}\right],\left[a_{1}, a_{2}\right], \ldots,\left[a_{i-2}, a_{i-1}\right]\right\}$ where $a_{v}$ is an endpoint of an element of $L_{t}$.

(b) $p<j$. Let

$$
\begin{aligned}
& L_{1}=\left\{\left[a_{i+1}, a_{i+2}\right], \ldots,\left[a_{p-2}, a_{p-1}\right]\right\} \\
& L_{2}=\left\{\left[a_{p+1}, a_{p+2}\right], \ldots,\left[a_{j-2}, a_{j-1}\right]\right\}
\end{aligned}
$$

and

$$
L_{3}=\left\{\left[a_{j+1}, a_{j+2}\right], \ldots,\left[a_{q-2}, a_{q-1}\right]\right\} .
$$

If each of $f\left(U_{1}^{*}\right), f\left(U_{2}^{*}\right)$ and $f\left(U_{3}^{*}\right)$ intersects one of $f\left(A_{2}^{*}\right)$ and $f\left(B_{2}^{*}\right)$, then proceed as in 4(a). If not, then $f\left(U_{1}^{*}\right)$ must be the one that fails to meet $f\left(A_{2}^{*}\right)$ or $f\left(B_{2}^{*}\right)$. Since $f$ is prime there exists $\left\{a_{v}, a_{w}\right\} \in W$ such that $i<v<q$ and $w<i$ or $q<w$. If $p<v<j$ or $j<v<q$ proceed as in 4(a). Now suppose $i<v<p$. By condition 1 of Theorem 19 of [9] there is an element $\left\{a_{t}, a_{u}\right\}$ of $W$ such that $u \neq j, p<u<q$, and $t<p$ or $t<q$. If $t>q$ or $t<i$ proceed as in 4(a). If $i<t<p$ let

$$
L=L_{1}+L_{m}+\left\{\left[a_{q+1}, a_{q+2}\right], \ldots,\left[a_{2 n}, a_{1}\right],\left[a_{1}, a_{2}\right], \ldots,\left[a_{i-2}, a_{i-1}\right]\right\}
$$


where $a_{u}$ is an endpoint of an element of $L_{m}$. This concludes cases 1-4 and shows that in any event the desired collection $L$ is obtained.

Suppose $f\left(L^{*}\right)$ intersects $f\left(A_{2}^{*}\right)$ and $f\left(A_{1}^{*}\right)$. There is an $\operatorname{arc} A B$ from point $A$ in $f\left(A_{1}^{*}\right) \cdot f\left(A_{n}\right)$ to a point $B$ in $f\left(A_{2}^{*}\right) \cdot f\left(A_{n}\right)$. There is a subarc $C D$ of $A B$ such that (1) $C$ and $D$ are in $f\left(A_{n}\right)$, and (2) $C$ belongs to one of $f\left(A_{1}^{*}\right)$ and $f\left(A_{2}^{*}\right)$ and $D$ belongs to the other and arc $C D$ misses $f\left(B_{2}^{*}\right)$ (or use $f\left(A_{1}^{*}\right)$ and $f\left(B_{2}^{*}\right)$ and require that $C D$ miss $f\left(A_{2}^{*}\right)$, or use $f\left(A_{2}^{*}\right)$ and $f\left(B_{1}^{*}\right)$ and require that $C D$ miss $f\left(B_{2}^{*}\right)$, or use $f\left(B_{2}^{*}\right)$ and $f\left(B_{1}^{*}\right)$ and require that $C D$ miss $\left.f\left(A_{2}^{*}\right)\right)$ and (3) $C D$ contains no proper subarc with the same property. Let $M=\left\{L^{\prime} \in L: f\left(L^{\prime}\right) \subset C D\right\}$. (It follows by Lemma 1 that $C D$ is the sum of such sets.)

Suppose for example that $C \in f\left(A_{1}^{*}\right)$ and $D \in f\left(A_{2}^{*}\right)$. The arc $C D$ and the arc $f\left(B_{1}^{*}\right)$ have endpoints that separate each other on Bd $U$. Therefore $C D$ and $f\left(B_{1}^{*}\right)$ intersect, a contradiction.

If $C \in f\left(A_{1}^{*}\right)$ and $D \in f\left(B_{2}^{*}\right)$ let $R$ denote the first point of $f\left(B_{1}^{*}\right)$ in the order $Q C P$ on $f\left(A_{1}^{*}\right)$. Let $N$ denote $\left\{L^{\prime} \in L: f\left(L^{\prime}\right) \subset\right.$ arc $\left.C R\right\}$. In this case $g\left(M^{*}\right)+g\left(N^{*}\right)$ and $g\left(A_{2}^{*}\right)$ are arcs whose endpoints separate each other on $\mathrm{Bd} V$. This involves a contradiction.

These two cases suffice to show how to handle the other two, so this concludes the proof of Theorem 1 .

Proof of Theorem 2. For each complementary domain $U$ of $\operatorname{Im} f$, let $U^{\prime}$ denote the unique complementary domain of $\operatorname{Im} g$ guaranteed by Theorem 1 . But starting with $\operatorname{Im} g$ and letting a $U^{\prime}$ correspond to $U^{\prime \prime}$ one must obtain the relation

$$
U \rightarrow U^{\prime} \rightarrow U^{\prime \prime}=U .
$$

Proof of Theorem 3. Suppose the complementary domain $U$ of $\operatorname{Im} f$ corresponds to the complementary domain $U^{\prime}$ of $\operatorname{Im} g$ under the correspondence guaranteed by Theorem 2. Let $B(U)$ denote the subcollection of $G\left(A_{n}\right)$ such that $f\left(B(U)^{*}\right)=\mathrm{Bd} U$. Of course, $g\left(B(U)^{*}\right)=\mathrm{Bd} U^{\prime}$. Also for $B_{1}, B_{2} \in B(U) f\left(B_{1}\right)$ intersects $f\left(B_{2}\right)$ if and only if $g\left(B_{1}\right)$ intersects $g\left(B_{2}\right)$. Remember also that for $\left\{a_{i}, a_{j}\right\} \in W, f\left(a_{i}\right)=f\left(a_{j}\right)$ and $g\left(a_{i}\right)=g\left(a_{j}\right)$.

By the Schoenflies Theorem there exist homeomorphisms $h_{1}$ and $h_{2}$ mapping $\bar{U}$ and $\bar{U}^{\prime}$, respectively, onto $T=\{z:|z| \leqq 1\}$ (for the case of the unbounded components use $T-\{0\})$. Define $h$ mapping $\bar{U}$ onto $\bar{U}^{\prime}$ by $h\left(h_{1}^{-1}(0)\right)=h_{2}^{-1}(0)$ and otherwise, for $P \in W-\{0\}$ let $t$ be a number in $[0,1)$ such that $P=r h_{1}(f(t))$ for some $r$ satisfying $0<r \leqq 1$ and define $h\left(h_{1}^{-1}(P)\right)$ to be $h_{2}^{-1}\left(r h_{2}(g(t))\right)$. Let $h$ be defined on all other $\bar{U}$ 's analogously. It is a simple matter to check that $h$ is a homeomorphism from $E^{2}$ onto $E^{2}$ such that $h f=g$. This concludes the proof of Theorem 3.

The collection $N\left(A_{n}\right)$ could also be defined for mappings into the two spheres, where the crossing of straight line intervals is replaced by the crossing of arcs (see $[4, \mathrm{p} .182])$. In this case Theorem 3 holds unrestricted in the sense that boundedness requirements simply disappear. 


\section{REFERENCES}

1. R. H. Crowell and R. H. Fox, Introduction to knot theory, Ginn, Boston, Mass., 1963.

2. C. F. Gauss, Werke (8), Teubner, Leipzig, 1900, 272, 282-286.

3. M. L. Marx, Normal curves arising from light open mappings of the annulus, Trans. Amer. Math. Soc. 120 (1965), 46-56.

4. R. L. Moore, Foundations of point set theory, Colloq. Publ., Vol. 13, Amer. Math. Soc., Providence, R. I., 1932.

5. J. v. Sz. Nagy, Über ein topologisches Problem von Gauss, Math. Zeit. 26 (1927), 579-592.

6. D. E. Penney, An algorithm for establishing isomorphism between tame prime knots in $E^{3}$, Doctoral Dissertation, Tulane University, New Orleans, La., 1965.

7. C. J. Titus, A theory of normal curves and some applications, Pacific J. Math. 10 (1960), 1083-1096.

8. - The combinatorial topology of analytic functions on the boundary of a disk, Acta Math. 106 (1961), 45-64.

9. L. B. Treybig, A characterization of the double point structure of the projection of a polygonal knot in regular position, Trans. Amer. Math. Soc. 130 (1968), 223-247.

TULANE UNIVERSITY,

New OrLeans, Louisiana 